\title{
Standard Million Population
}

National Cancer Institute

\section{Source}

National Cancer Institute. Standard Million Population. NCI Thesaurus. Code C71557.

A standard population for a geog raphic area consisting of a table giving the number of persons in each age group out of a theoretical cohort of 1,000,000 persons that is distributed by age in the same proportions as the population. 